\title{
The CUORE Data Acquisition System
}

\author{
S. Copello ${ }^{1}$ - S. Di Domizio ${ }^{2,3} \cdot$ A. Branca ${ }^{4,5} \cdot$ A. Caminata $^{3} \cdot$ L. Canonica $^{8}$. \\ A. Giachero $0^{6,7} \cdot$ E. Guardincerri ${ }^{3} \cdot$ L. Marini ${ }^{2,3,10} \cdot$ M. Pallavicini ${ }^{2,3} \cdot$ M. Vignati $^{9}$
}

Received: 18 August 2019 / Accepted: 24 November 2019 / Published online: 9 December 2019 (c) Springer Science+Business Media, LLC, part of Springer Nature 2019

\begin{abstract}
Large mass bolometers, thanks to their good energy resolution and high radiopurity, can be used for rare event searches, such as neutrinoless double beta decay or dark matter direct detection. The bolometric technique has been adopted by the CUORE experiment which is composed by an array of 988 tellurium dioxide bolometers with a total active mass of $741 \mathrm{~kg}$. The experiment started taking data in April 2017 at the Laboratori Nazionali del Gran Sasso (LNGS), Italy, with the scientific goal of searching for neutrinoless double beta decay of 130-Te. Given the increasing number of channels to be acquired, the readout chain became an important aspect of the construction of bolometer arrays. The CUORE data acquisition system here described, called Apollo, was initially developed for CUORE, but its high modularity and flexibility make it possible to use it also in other experiments, regardless of the specific characteristics of the setup such as the number of channels and the bolometer characteristics. Indeed, it has been used not only in CUORE but also in its predecessor CUORE-0, R\&D projects and upgrades such as CUPID-0.
\end{abstract}

Keywords CUORE - CUPID-0 - Bolometers · Data acquisition · Double beta decay

S. Copello

scopello@ge.infn.it

1 Gran Sasso Science Institute, 67100 L'Aquila, Italy

2 Dipartimento di Fisica, Università degli Studi di Genova, 16146 Genoa, Italy

3 INFN - Sezione di Genova, 16146 Genoa, Italy

4 Dipartimento di Fisica e Astronomia, Università di Padova, 35131 Padua, Italy

5 INFN - Sezione di Padova, 35131 Padua, Italy

6 Dipartimento di Fisica, Università di Milano-Bicocca, 20126 Milan, Italy

7 INFN - Sezione di Milano Bicocca, 20126 Milan, Italy

8 INFN - Laboratori Nazionali del Gran Sasso, 67100 Assergi, L'Aquila, Italy

9 INFN - Sezione di Roma, 00185 Rome, Italy

10 Department of Physics, University of California, Berkeley, CA 94720, USA

记 Springer 


\section{Introduction}

Bolometric detectors can be used for rare event searches [1], such as neutrinoless double beta decay $(0 \nu \beta \beta)$ [2] or dark matter interactions [3], where they are grouped in large arrays in order to increase the active mass of the experiments.

CUORE [4, 5] (Cryogenic Underground Observatory for Rare Events) is an excellent example of currently operating bolometric experiment. CUORE is an array of $988 \mathrm{TeO}_{2}$ crystals $(\sim 750 \mathrm{~g}$ each $)$ with a total active mass of $741 \mathrm{~kg}$. The experiment started taking data in April 2017 at the Laboratori Nazionali del Gran Sasso, Italy, with the search for $0 \nu \beta \beta$ of ${ }^{130} \mathrm{Te}$ as main scientific goal. Detectors are arranged in 19 modules, composed by 52 bolometers each. In order to improve the $0 \nu \beta \beta$ half-life sensitivity for the next generation experiment, several R\&D activities have being pursued in parallel using different isotopes [6, 7], increasing the source mass by means of isotope enrichment [8] or exploiting active particle discrimination techniques [9-15]. In this last case, light detectors are also needed to detect scintillation light or Cherenkov radiation. Given the requirement to operate at bolometric temperature, light detectors are obtained with secondary, lighter, bolometers capable of collecting the light emitted by the primary bolometer.

For both heat and light detectors, the energy E released by the interacting particle is evaluated by measuring the bolometer temperature variation. The bolometer temperature, around $10 \mathrm{mK}$, in CUORE is monitored by means of neutron transmutation-doped (NTD) germanium thermistors [16] glued on the crystal. Their resistance decreases as the temperature increases so, once biased with a constant current, an energy release appears as a voltage drop at its ends and typical values range from a few to hundreds of $\mu \mathrm{V} / \mathrm{MeV}$. Every detector is also equipped with a very stable silicon resistor $(R \sim 100 \mathrm{k} \Omega)$, glued on the crystal, used during data taking to inject a fixed amount of energy to emulate particle interactions $[17,18]$. Such heater signals are analyzed off-line to compensate thermal gain variations thus improving the energy resolution.

The readout chain operates in differential mode in order to suppress the common mode noise, and it is composed by three parts: low noise, programmable gain amplifier front ends, 6-pole anti-aliasing Bessel filters and the digitizers of the data acquisition (DAQ) system (more information on the readout chain can be found in [17, 19-22]). The DAQ system, called Apollo, has three main roles: digitize the signal, run the trigger algorithms and permanently store the acquired data to disks for the off-line analysis. Moreover, it controls the electronic devices composing the readout chain.

Thanks to its flexibility in terms of sampling frequency, number of channels and trigger algorithms, Apollo is currently used in several projects with different kinds of bolometers. An excellent example from this point of view is the CUPID-0 experiment, where ZnSe scintillating crystals are surrounded by thin germanium disks used as light detectors (LD) [10]. Pulses from different bolometers are reported in Fig. 1. 

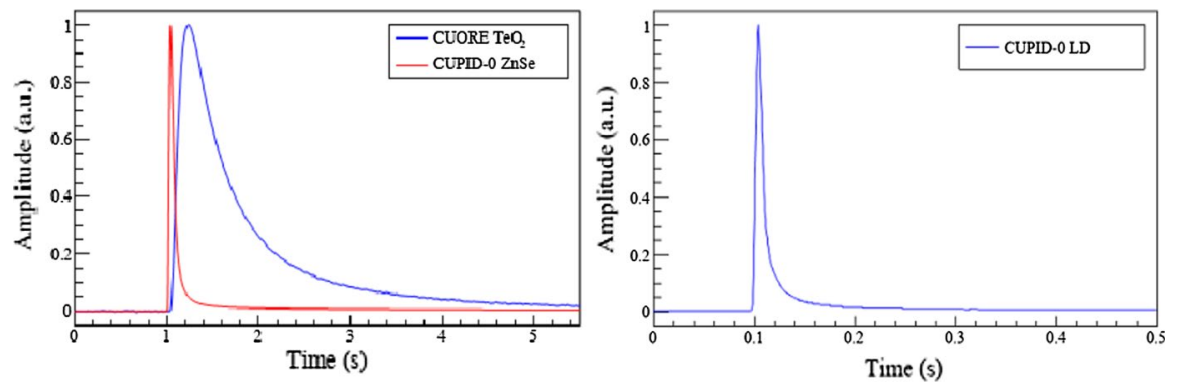

Fig. 1 Examples of pulses from CUORE and CUPID-0 experiments. The differences in time evolution require the acquisition parameters (e.g., sampling frequency and the window length) to be adaptable on a channel basis. Picture from [23] (Colour figure online)

\section{Data Acquisition Hardware}

The Apollo data acquisition system is based on National Instruments (NI) commercial digitizers, mainly NI-PXI-6284 even if also other boards have been used in smaller setups (e.g., NI-PXI-6133). NI-PXI-6284 [24] boards can acquire up to 16 channels in differential mode with an 18-bit ADC resolution and a programmable sampling frequency up to 500-k samples/s. An internal reference signal is used to correct gain and offset while an embedded analog circuitry allows to choose the input range from $\pm 0.1 \mathrm{~V}$ up to $\pm 10.5 \mathrm{~V}$, single or double ended, and to enable an anti-aliasing filter with cutoff frequency of $40 \mathrm{kHz}$ or $750 \mathrm{kHz}$. Additionally, several analog output channels and digital I/O lines are available.

The sampling frequency is selected according to the specific signal bandwidth which typically goes up to about $10 \mathrm{~Hz}$. In CUORE-0, $125 \mathrm{~Hz}$ was chosen because it was adequate compared to the signal time constants, while for CUORE $1 \mathrm{kHz}$ was selected for a more accurate pulse reconstruction. On the other hand, light detectors are faster and are usually digitized at $2 \mathrm{kHz}$. The ADC resolution is appropriate since it is negligible compared to the intrinsic detector resolution, respectively, $\sim 160 \mathrm{eV}$ compared to a few $\mathrm{keV}$ for heat detectors and less than $\sim 3 \mathrm{eV}$ compared to few tens of $\mathrm{eV}$ for light detectors.

Up to 17 digitizer boards can be enclosed in a single NI-PXI chassis that communicates via optical link to the data reader computer thanks to the controller module (PCIPXI-8336). The modularity of the system allows integrating several chassis in the same data acquisition. CUORE is the setup with the larger number of channel to be acquired, more than one thousand, corresponding to 64 digitizer boards hosted in six chassis. The PXI chassis clock is shared among the chassis thanks to the daisy chain connection.

Because of the incompatibility between the 8-channel NI connectors and the 12 channel D-Sub connectors coming from the Bessel filters, custom interface boards have been used to remap the signals. These Bessel-to-DAQ interface boards also allow accessing the I/O digital lines of every digitizer board.

Digitized data produced by every chassis are eventually transmitted via optical link to the corresponding computer where they are processed and stored. At the end of the run, data are automatically copied to two independent server farms and the original files are deleted from the DAQ computers. 


\section{Data Acquisition Software}

The Apollo software, sketched in Fig. 2, is composed of several independent processes, able to run on different computers and to communicate with each other over TCP/IP. All the software programs are written in the $\mathrm{C}++$ programming language taking advantage of the National Instrument and CERN ROOT libraries. The main process that manages the others in a coherent manner is called DaqServer, and it can be remotely driven, even taking advantage of a graphic user interface. The processes actually devoted to acquire the digital samples are called DataReader and run in parallel, one per each data reader computer (i.e., one per digitizer chassis). Every DataReader process reads the data stream from its chassis and applies the trigger algorithms on the channels therein. Indeed, given the low sampling frequency, no hardware trigger is needed while a software trigger is more flexible. Moreover more than one algorithm can be applied on the same channel. At least two triggers are usually used: One is called noise trigger and is a simple random trigger that provides the "empty events" used to monitor the detector stability and to evaluate average noise power spectra, and the second one is the signal trigger that fires in correspondence of a pulse and can be a derivative trigger or an optimum trigger (described in [25]).

After this step, the samples and the possible trigger flags are copied into some shared memories, one per channel, handled as circular buffers. A unique EventBuilder process accesses all the buffers, finds the trigger flags therein and stores on file the waveforms of the corresponding time windows. Per every trigger firing on a given channel, the EventBuilder saves also information coming from electrically coupled channels and from geometrically close bolometers. This will be used in the analysis for proper noise reduction strategy and for event coincidence evaluation. The EventBuilder usually runs on a dedicated computer, and a client-server mechanism is used to transfer the data from the data reader machine(s) to the event builder machine. Such mechanism is obtained via TCI/IP by two processes called MirrorServer and MirrorClient.

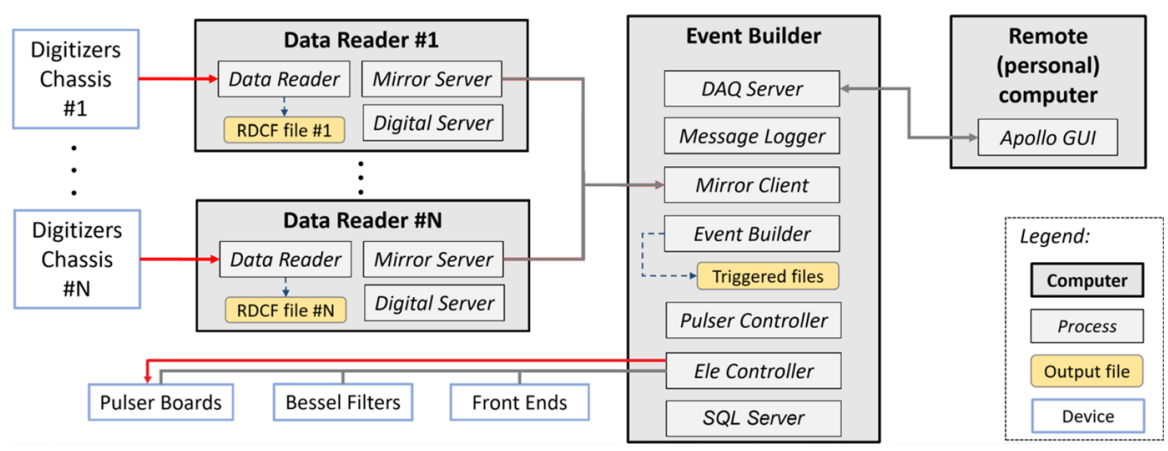

Fig. 2 Apollo software structure. Copies of the DataReader process, running on different computers, read the data from the digitizers while the DaqServer and other the ancillary processes run on a dedicated machine. Picture from [23] (Colour figure online) 
The relatively small data stream and the acquisition parallelization allow every DataReader to store on disk all the data coming from its chassis regardless the triggers. The resulting Raw Data Continuous Flow (RDCF) files can be used for more sophisticated off-line analysis or even to virtually repeat the experiment with, e.g., new trigger algorithms. This feature also allows not to save the waveforms into the regular file since they can be easily retrieved from the RDCF files, thus saving system resources and disk space.

A PostgreSQL database is used to store information about the channel and electronics mapping, bolometers characteristics and position, configuration of digitizers, triggers and event building parameters. It is also used to keep track of the acquired measurements (start and stop time, measurement type, etc.) and, by the off-line analysis routines, to store various non-event-based quantities.

In addition, some more ancillary processes are present in Apollo. The EleController process configures and monitors the status of the electronic devices of the apparatus. Here the communication interfaces include CAN bus, RS232 and GPIB. EleController is a multi-thread process that allows concurrent communication with different devices, thus reducing the detector configuration time. The PulserController drives the pulser boards to periodically fire thermal calibration pulses in the bolometers (these pulses are physically generated by the heaters glued on the crystals described in Sect. 1). At the same time, a digital signal is sent to the digitizers, via the I/O line on the NI boards, to tag such events. Finally, all the processes interact via TCP/IP with the MsgLogger which collects log messages from all the active processes running in the Apollo computers.

Besides the processes described above, a set of scripts and routines exist in Apollo; the most relevant ones perform specific tasks interacting with the detector (load curves measurement and working points definition [26]), update the database or configure the operating system of the computers of the system.

\section{Conclusions}

The DAQ system here described, Apollo, has been originally designed for the CUORE experiment and its predecessors. It is a scalable and adaptable system, even suitable for heterogeneous setups composed of heat and light detectors like CUPID0 . One of its key features is its hardware trigger-less operability, which makes it especially suitable for experiments where saving the whole detector waveforms is preferable. This approach allows the application of off-line application-specific triggers. In CUORE, Apollo has demonstrated its ability to handle the data of a tonscale experiment.

Acknowledgements This work was sponsored by the Istituto Nazionale di Fisica Nucleare (INFN) with the support of the DOE Office of Science, Office of Nuclear Physics, Contract No. DE-FG02-08ER41551. The authors thank the CUORE and CUPID-0 Collaborations, the directors and staff of the Laboratori Nazionali del Gran Sasso and the technical staff of Genova, INFN division. 


\section{References}

1. S. Pirro, P. Mauskopf, Ann. Rev. Nucl. Part. Sci. 67, 161-181 (2017)

2. O. Cremonesi, M. Pavan, Adv. High Energy Phys. 2014, 951432 (2014)

3. M. Klasen, M. Pohl, Prog. Part. Nucl. Phys. 85, 348 (2015)

4. C. Arnaboldi et al., Nucl. Instrum. Meth. A518, 775-798 (2004)

5. CUORE Collaboration, D.R. Artusa et al., Adv. High Energy Phys. 2015, 879871 (2015)

6. D.R. Artusa et al., Eur. Phys. J. C 76, 619 (2016)

7. I. Dafinei et al., J. Cryst. Growth 475, 158-170 (2017)

8. D.R. Artusa et al., Phys. Lett. B 767, 321-329 (2017)

9. E. Armengaud et al., Eur. Phys. J. C 77, 785 (2017)

10. J.W. Beeman et al., JINST 8, P05021 (2013)

11. L. Bergé et al., Phys. Rev. C 97, 032501 (2018)

12. M. Biassoni et al., Eur. Phys. J. C 75, 480 (2015)

13. M. Willers et al., JINST 10, P03003 (2015)

14. K. Schäffner et al., Astropart. Phys. 69, 44-49 (2015)

15. L. Cardani et al., Appl. Phys. Lett. 110, 033504 (2017)

16. N. Wang, F.C. Wellstood, B. Sadoulet, E.E. Haller, J. Beeman, Phys. Rev. B 41, 3761 (1990)

17. K. Alfonso, L. Cassina, A. Giachero, C. Gotti, G. Pessina, P. Carniti, JINST 13, P02029 (2018)

18. C. Arnaboldi, G. Pessina, E. Previtali, I.E.E.E. Trans, Nucl. Sci. 50, 979-986 (2003)

19. C. Arnaboldi, P. Carniti, L. Cassina, C. Gotti, X. Liu, M. Maino et al., JINST 13, P02026 (2018)

20. C. Arnaboldi, M. Cariello, S. Di Domizio, A. Giachero, G. Pessina, Nucl. Instrum. Method A 617, 327 (2010)

21. C. Arnaboldi, A. Baù, P. Carniti, L. Cassina, A. Giachero, C. Gotti et al., Rev. Sci. Instrum. 86, 124703 (2015)

22. P. Carniti, L. Cassina, C. Gotti, M. Maino, G. Pessina, Rev. Sci. Instrum. 87, 054706 (2016)

23. S. Di Domizio et al., JINST 13, P12003 (2018)

24. NI-PXI-6284 manual at http://www.ni.com/pdf/manuals/375219c.pdf

25. S. Di Domizio, F. Orio, M. Vignati, JINST 6, P02007 (2011)

26. CUORE Collaboration, C. Alduino et al., JINST 11, P09003 (2016)

Publisher's Note Springer Nature remains neutral with regard to jurisdictional claims in published maps and institutional affiliations. 